\title{
Association of NAT2 phenotype with risk of head and neck carcinoma: A meta-analysis
}

\author{
YOUYANG ZHENG ${ }^{1}$, YONG $\mathrm{LI}^{2}$, YAOSHU TENG ${ }^{2}$, ZHEN ZHANG $^{3}$ and XIAOLIN CAO ${ }^{2}$ \\ ${ }^{1}$ Department of Stomatology, The Second Affiliated Hospital, Zhejiang University, School of Medicine, Hangzhou 310009; \\ ${ }^{2}$ Department of Otolaryngology Head and Neck Surgery, Hangzhou First People's Hospital, Hangzhou 310006; \\ ${ }^{3}$ Department of Neurosurgery, Provincial Hospital Affiliated to Shandong University, Jinan 250021, P.R. China
}

Received August 18,2011; Accepted November 17, 2011

DOI: $10.3892 / \mathrm{ol} .2011 .493$

\begin{abstract}
N-acetyltransferase 2 (NAT2) is a key enzyme involved in the metabolism of xenobiotics and plays a significant role in the detoxification of numerous potential carcinogens. According to its acetylation status, NAT2 acetylator may be classified into two phenotypes, rapid and slow. Numerous studies have demonstrated that the polymorphisms of NAT2 were correlated with individual susceptibility to several malignant neoplasms, including head and neck carcinomas (HNC). However, the associations between the acetylator phenotypes and HNC risk in each study were not entirely consistent. To assess these associations more comprehensively, we performed a meta-analysis. In this meta-analysis, 16 eligible studies including 2,965 cases with HNC and 3,919 controls were identified by searching the databases of PubMed, Medline and the ISI Web of Knowledge. Odds ratios (ORs) with 95\% confidence intervals (95\% CIs) were used to evaluate the association. No significant associations between the rapid acetylator phenotype in NAT2 and HNC risk were found either in the overall analysis $\left(\mathrm{OR}=0.98 ; 95 \%\right.$ CI $\left.0.83,1.15 ; \mathrm{I}^{2}=57 \% ; \mathrm{P}_{\text {heterogeneity }}=0.003\right)$ or in the subgroup analysis by ethnicity (for the Caucasian population, $\mathrm{OR}=1.03,95 \% \mathrm{CI} 0.85,1.24, \mathrm{I}^{2}=63 \%, \mathrm{P}_{\text {heterogeneity }}=0.002$; for other mixed populations, $\mathrm{OR}=0.78,95 \% \mathrm{CI}=0.61,1.00, \mathrm{I}^{2}=0 \%$, $\left.\mathrm{P}_{\text {heterogeneity }}=0.47\right)$. In conclusion, this meta-analysis suggested that there is no association between the NAT2 phenotype and the risk of HNC.
\end{abstract}

\section{Introduction}

Head and neck carcinoma (HNC), arising from the sites of the oral cavity, oropharynx, hypopharynx and larynx, is the

Correspondence to: Dr Xiaolin Cao, Department of Otolaryngology Head and Neck Surgery, Hangzhou First People's Hospital, 261 Huansha Road, Hangzhou, Zhejiang 310006, P.R. China

E-mail: doctorcaoxiaolin@163.com

Abbreviations: NAT2, N-acetyltransferase 2; HNC, head and neck carcinoma; OR, odds ratio; $95 \% \mathrm{CI}, 95 \%$ confidence interval

Key words: N-acetyltransferase 2, phenotype, head and neck carcinoma sixth most common type of cancer worldwide (1). It is characterized by a moderately low survival rate, a high recurrence rate, and a high rate of second primary malignancy (2). In 2009, approximately 49,260 new cases (35,530 males and 13,730 females) of HNC were diagnosed in the USA, and 11,480 deaths occurred (8,300 males and 3,180 females) (1). Present evidence has proven that numerous factors contribute to the risk of squamous cell carcinoma of the head and neck, including tobacco use, alcohol consumption (3), viral infection (4) and genetic polymorphisms (5). In particular, tobacco smoking and alcohol consumption account for approximately $80 \%$ of HNC cases $(6,7)$. However, it is worth noting that only a fraction of smokers and alcohol consumers develop HNC. This phenomenon suggests that host factors, including genetic variation, contribute to the inter-individual variation in the susceptibility to HNC.

Numerous procarcinogens are known to exist in tobacco, including polycyclic aromatic hydrocarbons (PAHs), aromatic and heterocyclic amines; nitroso-compounds in smoking tobacco; and nitrosamines, aromatic and heterocyclic amines in smokeless tobacco. Most of these carcinogens generally undergo bioactivation and inactivation by phase I and II enzymes, respectively. The phase II enzymes, including Glutathione S-transferases (GSTs) and N-acetyltransferase (NAT), contribute to the detoxification metabolism. Human NAT comprises the isoenzymes, NAT1 and NAT2. In contrast to the wide tissue distribution of NAT1, NAT2 is expressed predominantly in liver (8), although it is also found in numerous human tissues (9). NAT2 is a key phase II enzyme that participates in the bioconversion of aromatic and heterocyclic amines $(10,11)$. According to the activity and stability that arise from its DNA sequence variations, the NAT2 gene may be classified into two allele types; slow and rapid (12). Previous studies demonstrated that either slow or rapid acetylation may be associated with various tobacco-associated cancers. Cascorbi et al reported that rapid acetylation status was a highly significant risk factor in lung cancer development (13). Inversely, Hein et al found that NAT2 slow acetylation increased the risk of bladder cancer (14). In other relevant studies, no significant association between NAT2 polymorphisms and cancer was found, including colon (15), gastric (16), breast (17) and liver (18). These findings indicated that the activities of the NAT2 enzyme may be associated with the type of cancer, and its 
variance may be due to inter-individual variation of genetic susceptibility to cancers in the general population.

HNC belongs to the tobacco-associated group of cancers; thus, NAT2 plays a key role in the development of these types of cancer. A number of molecular epidemiological studies investigated the correlation between acetylator phenotypes in NAT2 and the risk of HNC. However, until now the results have been inconsistent and even contradictory. Therefore, we performed a meta-analysis of 16 published studies including 2,965 cases and 3,919 controls to obtain a more precise evaluation of the association between the NAT2 acetylator phenotype and HNC risk.

\section{Materials and methods}

Literature search strategy. Eligible studies were identified by searching the databases of PubMed, Medline and the ISI Web of Knowledge for relevant literature in English. The latest searches were undertaken on July 5, 2011. The following queries were used: ['N-acetyltransferase 2' or 'NAT 2'], and ['polymorphism' or 'SNP' or 'phenotype'], and ['head and neck cancer' or 'oropharyngeal cancer' or 'hypopharyngeal cancer' or 'oral cancer' or 'laryngeal cancer']. All of the searched studies were retrieved and their references were checked carefully to find additional eligible studies.

Inclusion and exclusion criteria. To identify eligible studies in this meta-analysis, the following criteria were established: i) case-control studies investigating the association of the NAT2 acetylator phenotype with HNC risk; ii) the diagnosis of head and neck cancer, including oral, oropharyngeal, hypopharyngeal and laryngeal cancer was confirmed by pathological examination; iii) controls were confirmed as free from HNC; iv) the sample used in the studies was blood; v) the studies were full-text articles; and vi) the studies were written in English.

The exclusion criteria were: i) non-case-control studies; ii) cases with other types of cancer, including nasopharyngeal and thyroid cancer; iii) case reports, letters, reviews, editorial articles and non-full-text literature; iv) no available phenotype or genotype information; and v) duplication of the previous publications.

Data extraction. The full-texts of the candidate articles were reviewed carefully by two independent investigators. The following variables were extracted from each study if available: the first author's surname, publication year, country of origin and ethnicity of participants, and the number of cases and controls with the studied phenotypes. According to the data from the Louisville University website (http://www. louisville.edu/medschool/pharmacology/NAT.html, updated July 22, 2011), NAT2*4 is regarded as the most common rapid acetylation allele, and NAT2*11A, NAT2*12A-C,NAT2*13A and $N A T 2 * 18$ are also considered as rapid alleles. Other alleles are considered as slow. In our studies, the NAT2 acetylator phenotype was classified into two groups according to its acetylation status, rapid or slow. A rapid acetylator is defined as being a carrier of at least one of the rapid acetylator alleles, whereas the slow acetylator carries two slow acetylator alleles. The different ethnic descents were classified as Caucasian, Asian, Turkish or other. Data were extracted independently by two investigators. If the results were discordant, a third investigator checked the data.

Statistical analysis. The meta-analysis was performed using the Review Manager 5 (version 5.0.18, provided by the Cochrane Collaboration). The strength of the association between the NAT2 acetylator phenotype and individual susceptibility to HNC was estimated using odds ratios (ORs) with $95 \%$ confidence intervals (95\% CIs). The pooled ORs were evaluated for rapid versus slow acetylator phenotypes. To evaluate the ethnicity-specific effect, subgroup analyses were performed according to ethnic group (Caucasian and other mixed populations). $\mathrm{P} \leq 0.05$ was considered to indicate statistical significance.

The heterogeneity among studies was estimated using Chi-square-based Q-tests. We also quantified the effect of heterogeneity by the $\mathrm{I}^{2}$ test. The $\%$ CI ranges from 0 to $100 \%$ and represents the proportion of inter-study variability that may be attributed to heterogeneity rather than chance. \% CI values of 25,50 and $75 \%$ were defined as low, moderate and high estimates, respectively. When the Q-test P-value was $>0.1$ or the $\%$ CI $<50 \%$, ORs were pooled according to the fixed-effects model (Mantel-Haenszel model). Otherwise, the random-effects model (DerSimonian and Laird model) was used. One-way sensitivity analyses were used to assess the stability of the meta-analysis results.

The Begg's funnel plots and Egger's test were conducted to evaluate the possible publication bias using Stata (version 11; StataCorp, College Station, TX, USA). Statistical significance was considered when the P-value of Egger's test was $\leq 0.05$.

P-values were two-sided. Two reviewers analyzed data independently and obtained the same results.

\section{Results}

Study characteristics. The process of study selection and exclusion is shown in Fig. 1. A total of 45 abstracts were retrieved through PubMed and Medline, and 2 additional articles were found through the ISI Web of Knowledge. After reviewing all 47 articles carefully, 16 eligible articles including 2,965 cases with HNC and 3,919 controls were identified and included in the final meta-analysis (19-34). The main characteristics of all 16 studies are described in Table I. The studies were case-control studies that evaluated the association between the NAT2 acetylation status and the susceptibility to HNC. The year of publication of the included studies ranged from 1998 to 2010. There were 11 studies of Caucasians (19,21-23,25-28,30,31,34), 3 of Asians (24,32,33), 1 of Turkish (20) and 1 of a mixed population (including Caucasian and other mixed populations) (29). All cases of HNC were pathologically confirmed. All articles were written in English.

Meta-analysis. The pooled ORs were evaluated for rapid versus slow acetylator phenotypes in NAT2. The overall OR was 0.98 and the $95 \% \mathrm{CI}$ was $0.83,1.15(\mathrm{P}=0.76)$. In the subgroup analysis of ethnicity, no increased risks were found either in the Caucasian population $(\mathrm{OR}=1.03$; $95 \% \mathrm{CI} 0.85$, $1.24 ; \mathrm{P}=0.37)$ or in the other mixed populations $(\mathrm{OR}=0.78$; 95\% CI 0.61, 1.00; $\mathrm{P}=0.05$ ) (Table II). 
Table I. Main characteristics of all studies included in the meta-analysis.

\begin{tabular}{|c|c|c|c|c|c|c|c|}
\hline \multirow[t]{2}{*}{ First author (Refs.) } & \multirow{2}{*}{$\begin{array}{c}\text { Publication } \\
\text { year }\end{array}$} & \multirow{2}{*}{$\begin{array}{l}\text { Country } \\
\text { of origin }\end{array}$} & \multirow[t]{2}{*}{ Ethnicity } & \multicolumn{2}{|c|}{ Cases } & \multicolumn{2}{|c|}{ Controls } \\
\hline & & & & Rapid & Slow & Rapid & Slow \\
\hline Chatzimichalis (19) & 2010 & Greece & Caucasian & 49 & 39 & 37 & 65 \\
\hline Demokan (20) & 2010 & Turkey & Turkish & 45 & 50 & 48 & 45 \\
\hline $\operatorname{Buch}(21)$ & 2008 & USA & Caucasian & 98 & 84 & 175 & 224 \\
\hline Harth (22) & 2008 & Germany & Caucasian & 123 & 189 & 119 & 181 \\
\hline Boccia (23) & 2008 & Italy & Caucasian & 101 & 109 & 117 & 128 \\
\hline Majumder (24) & 2007 & India & Asian & 107 & 190 & 137 & 205 \\
\hline Rydzanicz (25) & 2005 & Poland & Caucasian & 93 & 89 & 71 & 72 \\
\hline Gajecka (26) & 2005 & Poland & Caucasian & 162 & 127 & 146 & 165 \\
\hline Hahn (27) & 2002 & Germany & Caucasian & 35 & 59 & 35 & 57 \\
\hline Varzim (28) & 2002 & Portugal & Caucasian & 41 & 47 & 96 & 76 \\
\hline \multirow[t]{2}{*}{ Chen (29) } & 2001 & USA & Caucasian & 133 & 187 & 226 & 294 \\
\hline & & & Others (mixed) & 10 & 11 & 24 & 8 \\
\hline \multicolumn{8}{|l|}{ Jourenkova } \\
\hline -Mironova (30) & 1999 & France & Caucasian & 108 & 142 & 81 & 91 \\
\hline Henning (31) & 1999 & Germany & Caucasian & 117 & 138 & 224 & 286 \\
\hline Morita (32) & 1999 & Japan & Asian & 127 & 18 & 147 & 17 \\
\hline Katoh (33) & 1998 & Japan & Asian & 55 & 7 & 115 & 7 \\
\hline Gonzalez (34) & 1998 & Spain & Caucasian & 47 & 28 & 163 & 37 \\
\hline
\end{tabular}

Rapid, rapid acetylator phenotype; Slow, slow acetylator phenotype.

Table II. Summary of ORs with $95 \%$ CIs for rapid acetylator phenotype in NAT2 and head and neck carcinoma risk.

\begin{tabular}{|c|c|c|c|}
\hline First author (Refs.) & Ethnicity & OR & $95 \% \mathrm{CI}$ \\
\hline Chatzimichalis (19) & Caucasian & 2.21 & $1.23,3.95$ \\
\hline Demokan (20) & Turkish & 0.84 & $0.48,1.50$ \\
\hline Buch (21) & Caucasian & 1.49 & $1.05,2.12$ \\
\hline Harth (22) & Caucasian & 0.99 & $0.72,1.37$ \\
\hline Boccia (23) & Caucasian & 1.01 & $0.70,1.47$ \\
\hline Majumder (24) & Asian & 0.84 & $0.61,1.16$ \\
\hline Rydzanicz (25) & Caucasian & 1.06 & $0.68,1.64$ \\
\hline Gajecka (26) & Caucasian & 1.44 & $1.04,1.99$ \\
\hline Hahn (27) & Caucasian & 0.97 & $0.53,1.75$ \\
\hline Varzim (28) & Caucasian & 0.69 & $0.41,1.16$ \\
\hline \multirow[t]{3}{*}{ Chen (29) } & Caucasian & 0.93 & $0.70,1.23$ \\
\hline & Mixed & 0.3 & $0.09,0.98$ \\
\hline & Total & 0.87 & $0.66,1.15$ \\
\hline $\begin{array}{l}\text { Jourenkova } \\
\text {-Mironova (30) }\end{array}$ & Caucasian & 0.85 & $0.58,1.26$ \\
\hline Henning (31) & Caucasian & 1.08 & $0.80,1.46$ \\
\hline Morita (32) & Asian & 0.82 & $0.40,1.65$ \\
\hline Katoh (33) & Asian & 0.48 & $0.16,1.43$ \\
\hline \multirow[t]{4}{*}{ Gonzalez (34) } & Caucasian & 0.38 & $0.21,0.69$ \\
\hline & Total Caucasian & 1.03 & $0.85,1.24$ \\
\hline & Mixed & 0.78 & $0.61,1.00$ \\
\hline & Total & 0.98 & $0.83,1.15$ \\
\hline
\end{tabular}

OR, odds ratio; CI, confidence interval.

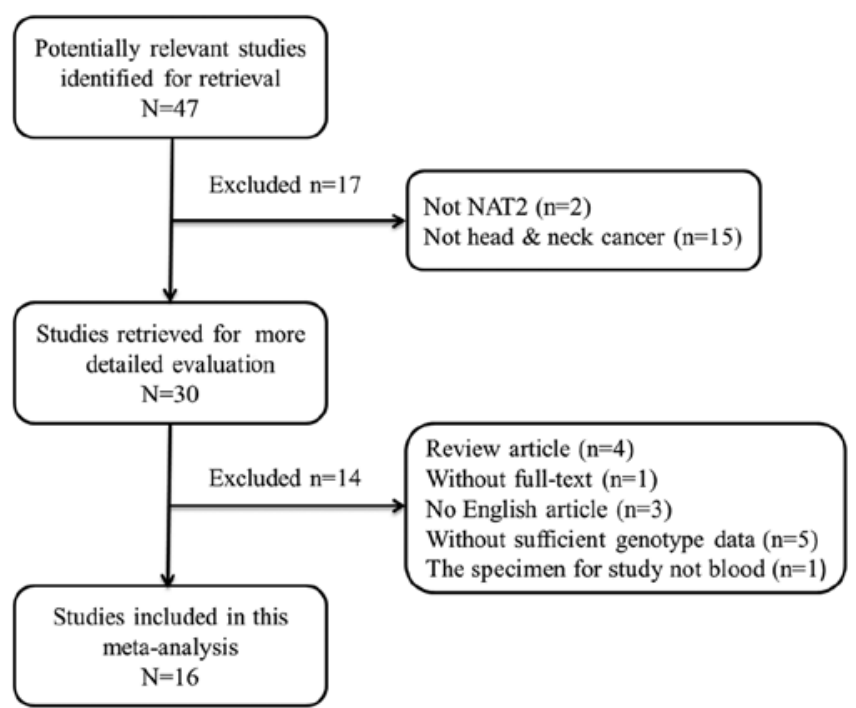

Figure 1. Study flow chart, showing the selection of case-control studies.

Heterogeneity and sensitivity analysis. Heterogeneity among all studies was assessed both in the overall comparisons and in the subgroup analyses. The evidence of significant heterogeneity was found in the overall comparison $\left(\mathrm{P}_{\text {heterogeneity }}=0.003\right.$, $\% \mathrm{CI}=57 \%)$ and the Caucasian subgroup $\left(\mathrm{P}_{\text {heterogeneity }}=0.002\right.$, $\% \mathrm{CI}=63 \%$ ), whereas no significant heterogeneity was observed in the other mixed population subgroup $\left(\mathrm{P}_{\text {heterogeneity }}=0.47\right.$, $\% \mathrm{CI}=0 \%$ ) (Fig. 2).

In the sensitivity analysis, the effect of each study on the pooled OR was examined by repeating the meta-analysis while 


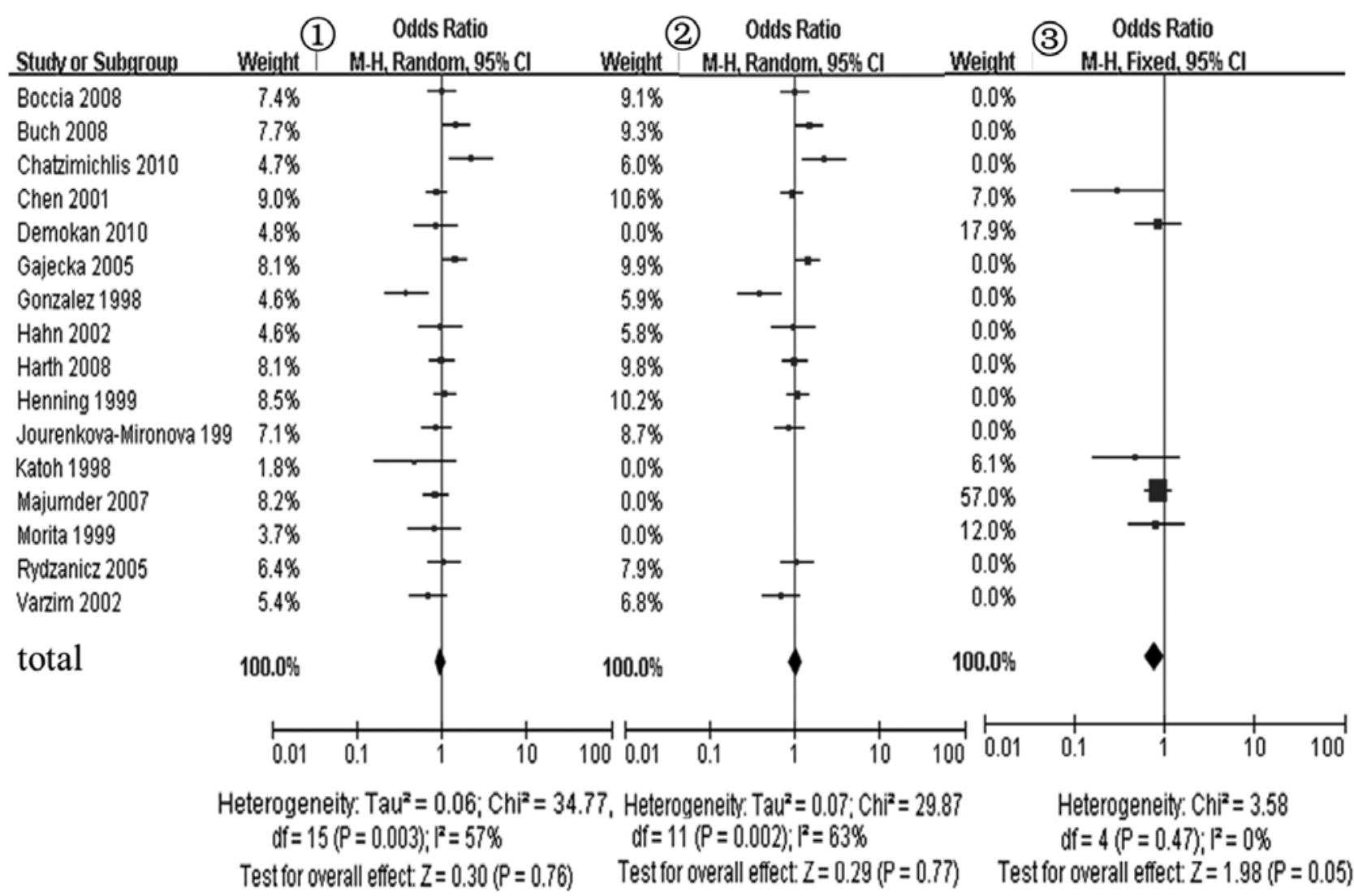

Figure 2. Forest plot showing the association between the rapid acetylator phenotype in NAT2 and risk of head and neck carcinoma. (1), in overall population; (2), in Caucasian population; (3, in other population.

omitting each study, one at a time. This procedure proved that our results were reliable and robust.

Publication bias. Begg's funnel plots and Egger's tests were conducted to graphically estimate the publication bias of the included literature. The shape of funnel plots revealed no obvious asymmetry. In addition, Egger's test also indicated no significant publication bias in this meta-analysis $(\mathrm{t}=-1.09, \mathrm{P}=0.293)$.

\section{Discussion}

As a well-known environmental risk factor, tobacco contains numerous potential carcinogens, including PAHs, which require bioactivation or inactivation via a series of enzymes. To the best of our knowledge, $\mathrm{N}$-acetylation is a crucial step during the activation of tobacco-related carcinogens (35), therefore tobacco-associated cancers may be associated with the NAT enzyme. Human N-acetyltransferase has two subtypes, NAT1 and NAT2. The NAT2 gene is located on the chromosome 8p21.3-23.1 and 8p22, and it encodes a 290-amino-acid protein $(35,36)$. As an essential phase II enzyme, NAT2 is key in the process of cancer development. However, the carcinogenesis of tobacco is relatively complex and the role of NAT2 described in different studies is not entirely consistent. In the studies concerning the association of NAT2 polymorphisms with the increased risk of gastric cancer, Shen et al (37) indicated that the rapid acetylator was the protective factor for the development of gastric cancer, whereas Ladero et al (38) suggested that it was regarded as a risk factor. However, results in other relevant studies demonstrated that no significant association between NAT2 polymorphisms and gastric cancer was found $(37,39,40)$. This discrepancy has also existed in the studies of other tobacco-associated cancers, including lung cancer (41-43) and prostate cancer (44-46). Similar results were also found in the studies of HNC, which is strongly associated with tobacco smoking (6). In their study, Gara et al indicated that the T341C mutation of the NAT2 gene (slow allele) was associated with an elevated risk of HNC in the Tunisian population (47). Unal et al (48) and Lei et al (49) also found that the NAT2 slow acetylator phenotype may increase the risk of susceptibility to laryngeal carcinoma. Among the 16 studies included in this meta-analysis, 2 studies suggested that the NAT2 rapid phenotype was associated with the risk of $\operatorname{HNC}(19,20)$, whereas 1 study suggested that it acted as a protective factor for HNC (34). In the remaining 13 studies, no significant association was found between NAT2 phenotypes and cancer risk. These results may be attributable to inter-individual variation (including ethnicity, and the status and level of smoking and alcohol consumption), and may also be due to the limitation of the sample size of these studies. Our meta-analysis included 2,965 cases with HNC and 3,919 controls, and we found no significant association between the NAT2 phenotype and HNC risk. The findings indicated that variations of NAT2 acetylator phenotype may not affect the development of HNC.

The NAT2 gene has several polymorphisms; at present, 66 alleles have been described (http://www.louisville.edu/ 
medschool/pharmacology/NAT.html, updated on July 22, 2011). The distributive proportion of NAT2 acetylator phenotypes varies in populations according to ethnicity and geographic origin. The percentage of slow acetylators is $40-70 \%$ in Europe, approximately $10 \%$ in Japan, 5\% in Eskimos and $87 \%$ in Egyptians (9). This suggested that NAT2 play different roles in the development of various types of cancer among different ethnic groups. However, in our meta-analysis, we found no significant association between the NAT2 rapid acetylator phenotype and the risk of HNC in either the Caucasian or other mixed population.

Although our meta-analysis pooled 16 relevant studies and the combined results may be more precise than each study individually, several limitations of this meta-analysis should be addressed. Firstly, although 16 studies were included with 2,965 cases and 3,919 controls, the number of studies remained insufficient and the sample size was relatively small, particularly for stratified analysis. This may have limited our ability to detect a certain degree of association. Secondly, in the subgroup analysis of ethnicity, the included studies involved only Caucasian, Asian and Turkish populations. For Asians, only 525 cases and 660 controls were used and for Turkish, only 1 study (involving 95 cases and 93 controls) was conducted. These numbers do not provide sufficient statistical power to evaluate the effect of the NAT2 phenotype on HNC risk in different ethnicities. Thirdly, heterogeneity was found among studies. We applied a random-effect model, but this did not exclude the effect of heterogeneity between studies, even though its effect may have been limited. Finally, although the selection of cases and controls in each study was well defined with the same inclusion and exclusion criteria, the potential confounding factors that affected our results may not have been taken into account. In spite of these limitations, our meta-analysis pooled the number of cases and controls from different studies and significantly increased the statistical power of the analysis.

In conclusion, this meta-analysis, including 16 case-control studies, suggests that there is no significant association between the NAT2 phenotype and the increased risk of HNC. Since few studies are available in this field, the results of this study should be further confirmed by single large sample case-control studies with well-designed, well-matched controls, particularly in populations other than Caucasian. In addition, the correlation between the NAT2 phenotype and HPV-associated HNC should be investigated.

\section{Acknowledgements}

We are indebted to the authors of the primary studies.

\section{References}

1. Jemal A, Siegel R, Xu J and Ward E: Cancer Statistics. CA Cancer J Clin 60: 277-300, 2010.

2. Ferlay J, Bray F, Pisani P and Parkin D: GLOBOCAN 2002: Cancer incidence, mortality and prevalence worldwide. IARC Press, Lyon, 2004.

3. Blot WJ, McLaughlin JK, Winn DM, et al: Source smoking and drinking in relation to oral and pharyngeal cancer. Cancer Res 48: 3282-3287, 1988

4. Dahlstrom KR, Adler-Storthz K, Etzel CJ, et al: Human papillomavirus type 16 infection and squamous cell carcinoma of the head and neck in never-smokers: a matched pair analysis. Clin Cancer Res 9: 2620-2626, 2003.
5. Sturgis EM and Wei Q: Genetic susceptibility - molecular epidemiology of head and neck cancer. Curr Opin Oncol 14: 310-317, 2002.

6. Vineis P, Alavanja M, Buffler P, et al: Tobacco and cancer: recent epidemiological evidence. J Natl Cancer Inst 96: 99-106, 2004.

7. Franceschi S, Talamini R, Barra S, et al: Smoking and drinking in relation to cancers of the oral cavity, pharynx, larynx, and esophagus in northern Italy. Cancer Res 50: 6502-6507, 1990.

8. Grant DM, Blum M, Beer M and Meyer UA: Monomorphic and polymorphic human arylamine $\mathrm{N}$-acetyltransferases: a comparison of liver isozymes and expressed products of two cloned genes. Mol Pharmacol 39: 184-191, 1991.

9. Blum M, Demierre A, Grant DM, Heim M and Meyer UA: Molecular mechanism of slow acetylation of drugs and carcinogens in humans. Proc Natl Acad Sci USA 88: 5237-5241, 1991.

10. Smith CA, Smith G and Wolf CR: Genetic polymorphisms in xenobiotic metabolism. Eur J Cancer 30A: 1921-1935, 1994.

11. Hein DW: Acetylator genotype and arylamine-induced carcinogenesis. Biochim Biophys Acta 948: 37-66, 1998.

12. Butcher NJ, Boukouvala S, Sim E and Minchin RF: Pharmacogenetics of the arylamine $\mathrm{N}$-acetyltransferases. Pharmacogenomics J 2: 30-42, 2002.

13. Cascorbi I, Brockmoller J, Mrozikiewicz PM, Bauer S, Loddenkemper R and Roots I: Homozygous rapid arylamine $\mathrm{N}$-acetyltransferase (NAT2) genotype as a susceptibility factor for lung cancer. Cancer Res 56: 3961-3966, 1996.

14. Hein DW: N-acetyltransferase 2 genetic polymorphism: effects of carcinogen and haplotype on urinary bladder cancer risk. Oncogene 25: 1649-1658, 2006.

15. Chen J, Stampfer MJ, Hough HL, et al: A prospective study of $\mathrm{N}$-acetyltransferase genotype, red meat intake, and risk of colorectal cancer. Cancer Res 58: 3307-3311, 1998.

16. Katoh $\mathrm{T}$, Boissy $\mathrm{R}$, Nagata $\mathrm{N}$, et al: Inherited polymorphism in the N-acetyltransferase 1 (NAT1) and 2 (NAT2) genes and susceptibility to gastric and colorectal adenocarcinoma. Int J Cancer 85: 46-49, 2000.

17. Lincz LF, Kerridge I, Scorgie FE, Bailey M, Enno A and Spencer A: Xenobiotic gene polymorphisms and susceptibility to multiple myeloma. Haematologica 89: 628-629, 2004.

18. Zhang XF, Bian JC, Zhang XY, et al: Are polymorphisms of $\mathrm{N}$-acetyltransferase genes susceptible to primary liver cancer in Luoyang, China? World J Gastroenterol 11: 1457-1462, 2005.

19. Chatzimichalis M, Xenellis J, Tzagaroulakis A, et al: GSTT1, GSTM1, GSTM3 and NAT2 polymorphisms in laryngeal squamous cell carcinoma in a Greek population. J Laryngol Otol 124: 318-323, 2010.

20. Demokan S, Suoglu Y, Gözeler M, Demir D and Dalay N: $\mathrm{N}$-acetyltransferase 1 and 2 gene sequence variants and risk of head and neck cancer. Mol Biol Rep 37: 3217-3226, 2010.

21. Buch SC, Nazar-Stewart V, Weissfeld JL and Romkes M: Casecontrol study of oral and oropharyngeal cancer in whites and genetic variation in eight metabolic enzymes. Head Neck 30: 1139-1147, 2008

22. Harth V, Schafer M, Abel J, et al: Head and neck squamous-cell cancer and its association with polymorphic enzymes of xenobiotic metabolism and repair. J Toxicol Environ Health A 71: 887-897, 2008.

23. Boccia S, Cadoni G, Sayed-Tabatabaei FA, et al: CYP1A1, CYP2E1, GSTM1, GSTT1, EPHX1 exons 3 and 4, and NAT2 polymorphisms, smoking, consumption of alcohol and fruit and vegetables and risk of head and neck cancer. J Cancer Res Clin Oncol 134: 93-100, 2008.

24. Majumder M, Sikdar N, Ghosh S and Roy B: Polymorphisms at XPD and XRCC1 DNA repair loci and increased risk of oral leukoplakia and cancer among NAT2 slow acetylators. Int J Cancer 120: 2148-2156, 2007.

25. Rydzanicz M, Wierzbicka M, Gajecka M, Szyfter W and Szyfter K: The impact of genetic factors on the incidence of multiple primary tumors (MPT) of the head and neck. Cancer Lett 224: 263-278, 2005.

26. Gajecka M, Rydzanicz M, Jaskula-Sztul R, Kujawski M, Szyfter W and Szyfter K: CYP1A1, CYP2D6, CYP2E1, NAT2, GSTM1 and GSTT1 polymorphisms or their combinations are associated with the increased risk of the laryngeal squamous cell carcinoma. Mutat Res 574: 112-123, 2005.

27. Hahn M, Hagedorn G, Kuhlisch E, Schackert HK and Eckelt U: Genetic polymorphisms of drug-metabolizing enzymes and susceptibility to oral cavity cancer. Oral Oncol 38: 486-490, 2002. 
28. Varzim G, Monteiro E, Silva R, Pinheiro C and Lopes C: Polymorphisms of arylamine $\mathrm{N}$-acetyltransferase (NAT1 and NAT2) and larynx cancer susceptibility. ORL J Otorhinolaryngol Relat Spec 64: 206-212, 2002.

29. Chen C, Ricks S, Doody DR, Fitzgibbons ED, Porter PL and Schwartz SM: N-Acetyltransferase 2 polymorphisms, cigarette smoking and alcohol consumption, and oral squamous cell cancer risk. Carcinogenesis 22: 1993-1999, 2001.

30. Jourenkova-Mironova N, Wikman $\mathrm{H}$, Bouchardy C, et al: Role of arylamine N-acetyltransferase 1 and 2 (NAT1 and NAT2) genotypes in susceptibility to oral/pharyngeal and laryngeal cancers. Pharmacogenetics 9: 533-537, 1999.

31. Henning S, Cascorbi I, Münchow B, Jahnke V and Roots I: Association of arylamine N-acetyltransferases NAT1 and NAT2 genotypes to laryngeal cancer risk. Pharmacogenetics 9: 103-111, 1999.

32. Morita S, Yano M, Tsujinaka T, et al: Genetic polymorphisms of drug-metabolizing enzymes and susceptibility to head-and-neck squamous-cell carcinoma. Int J Cancer 80: 685-688, 1999.

33. Katoh T, Kaneko S, Boissy R, Watson M, Ikemura K and Bell DA: A pilot study testing the association between $\mathrm{N}$-acetyltransferases 1 and 2 and risk of oral squamous cell carcinoma in Japanese people. Carcinogenesis 19: 1803-1807, 1998

34. González MV, Alvarez V, Pello MF, Menéndez MJ, Suárez C and Coto E: Genetic polymorphism of N-acetyltransferase-2, glutathione S-transferase-M1, and cytochromes P450IIE1 and P450IID6 in the susceptibility to head and neck cancer. J Clin Pathol 51: 294-298, 1998.

35. Hein DW, Doll MA, Fretland AJ, et al: Molecular genetics and epidemiology of the NAT1 and NAT2 acteylation polymorphisms. Cancer Epidemiol Biomarkers Prev 9: 29-42, 2000.

36. Vatsis KP, Martell Kj and Weber WW: Diverse point mutations in the human gene for polymorphic $\mathrm{N}$-acetyltranferase. Proc Natl Acad Sci USA 88: 6333-6337, 1991

37. Shen X, Zhang J, Yan Y, Yang Y, Fu G and Pu Y: Analysis and estimates of the attributable risk for environmental and genetic risk factors in gastric cancer in a Chinese population. J Toxicol Environ Health A 72: 759-766, 2009.

38. Ladero JM, Agúndez JA, Olivera M, et al: N-acetyltransferase 2 single-nucleotide polymorphisms and risk of gastric carcinoma. Eur J Clin Pharmacol 58: 115-118, 2002.
39. Zhang YW, Eom SY, Kim YD, et al: Effects of dietary factors and the NAT2 acetylator status on gastric cancer in Koreans. Int J Cancer 125: 139-145, 2009.

40. Boccia S, Sayed-Tabatabaei FA, Persiani R, et al: Polymorphisms in metabolic genes, their combination and interaction with tobacco smoke and alcohol consumption and risk of gastric cancer: a case-control study in an Italian population. BMC Cancer 7: 206, 2007.

41. Chiou HL, Wu MF, Chien WP, et al: NAT2 fast acetylator genotype is associated with an increased risk of lung cancer among never-smoking women in Taiwan. Cancer Lett 223: 93-101, 2005 .

42. Zupa A, Sgambato A, Bianchino G, et al: GSTM1 and NAT2 polymorphisms and colon, lung and bladder cancer risk: a casecontrol study. Anticancer Res 29: 1709-1714, 2009.

43. Borlak J and Reamon-Buettner SM: N-acetyltransferase 2 (NAT2) gene polymorphisms in colon and lung cancer patients. BMC Med Genet 7: 58, 2006

44. Hamasaki T, Inatomi $\mathrm{H}$, Katoh $\mathrm{T}$, et al: $\mathrm{N}$-acetyltransferase-2 gene polymorphism as a possible biomarker for prostate cancer in Japanese men. Int J Urol 10: 167-173, 2003.

45. Hein DW, Leff MA, Ishibe N, et al: Association of prostate cancer with rapid $\mathrm{N}$-acetyltransferase 1 (NAT1*10) in combination with slow N-acetyltransferase 2 acetylator genotypes in a pilot casecontrol study. Environ Mol Mutagen 40: 161-167, 2002.

46. Iguchi T, Sugita S, Wang CY, et al: MnSOD genotype and prostate cancer risk as a function of NAT genotype and smoking status. In Vivo 23: 7-12, 2009.

47. Gara S, Abdennebi M, Chatti S, Touati S, Ladgham A and Guemira F: Association of NAT2 gene substitution mutation T341C with increased risk for head and neck cancer in Tunisia. Acta Oncol 46: 834-837, 2007.

48. Unal M, Tamer L, Akbas Y, et al: Genetic polymorphism of $\mathrm{N}$-acetyltransferase 2 in the susceptibility to laryngeal squamous cell carcinoma. Head Neck 27: 1056-1060, 2005.

49. Lei D, Pan X, Guo C, et al: Relationship between polymorphism of $\mathrm{N}$-acetyltransferase 2 and genetic susceptibility to laryngeal carcinoma. Zhonghua Zhong Liu Za Zhi 24: 154-156, 2002. 